\title{
ARTICLE
}

\section{ECT2 associated to PRICKLE1 are poor-prognosis markers in triple-negative breast cancer}

\author{
Avais M. Daulat ${ }^{1}$, Pascal Finetti ${ }^{2}$, Diego Revinski ${ }^{1,3}$, Mônica Silveira Wagner ${ }^{1}$, Luc Camoin ${ }^{4}$, Stéphane Audebert ${ }^{4}$, Daniel Birnbaum ${ }^{2}$, \\ Laurent Kodjabachian ${ }^{3}$, Jean-Paul Borg ${ }^{1,4}$ and François Bertucci ${ }^{2}$
}

BACKGROUND: Triple-negative breast cancers (TNBC) are poor-prognosis tumours candidate to chemotherapy as only systemic treatment. We previously found that PRICKLE1, a prometastatic protein involved in planar cell polarity, is upregulated in TNBC. We investigated the protein complex associated with PRICKLE1 in TNBC to identify proteins possibly involved in metastatic dissemination, which might provide new prognostic and/or therapeutic targets.

METHODS: We used a proteomic approach to identify protein complexes associated with PRICKLE1. The mRNA expression levels of the corresponding genes were assessed in 8982 patients with invasive primary breast cancer. We then characterised the molecular interaction between PRICKLE1 and the guanine nucleotide exchange factor ECT2. Finally, experiments in Xenopus were carried out to determine their evolutionarily conserved interaction.

RESULTS: Among the PRICKLE1 proteins network, we identified several small G-protein regulators. Combined analysis of the expression of PRICKLE1 and small G-protein regulators had a strong prognostic value in TNBC. Notably, the combined expression of $E C T 2$ and PRICKLE1 provided a worst prognosis than PRICKLE1 expression alone in TNBC. PRICKLE1 regulated ECT2 activity and this interaction was evolutionary conserved.

CONCLUSIONS: This work supports the idea that an evolutionarily conserved signalling pathway required for embryogenesis and activated in cancer may represent a suitable therapeutic target.

British Journal of Cancer (2019) 120:931-940; https://doi.org/10.1038/s41416-019-0448-z

\section{BACKGROUND}

Triple-negative breast cancer is the most aggressive molecular subtype of breast cancer. ${ }^{1}$ In contrast with mammary cancers of other subtypes (hormone receptor-positive HR+/HER2-, and HER2 +), TNBCs do not express hormone receptors nor the HER2 oncogene, and thus are not candidates for hormone and antiHER2 therapy. ${ }^{1}$ Chemotherapy is the only systemic therapy currently approved for this subtype. However, TNBC is highly invasive with strong metastatic propensity. ${ }^{1}$ We recently identified PRICKLE1 as a poor-prognosis marker in breast cancer. ${ }^{2}$ PRICKLE1 is a member of a conserved group of proteins involved in planar cell polarity (PCP) pathway. ${ }^{3}$ This pathway is well characterised in epithelial tissue morphogenesis during embryonic development of invertebrates and vertebrates. The organisation of PCP relies on the spatial distribution of proteins at the plasma membrane such as Wnts, Frizzled, Van Gogh, Flamingo, Dishevelled, Diego, and Prickle. In vertebrates, homologous genes are involved in the regulation of convergent-extension (CE) during the early stages of gastrulation which leads to the organisation of cells to generate the head-to-tail axis. ${ }^{3,4}$ Prickle1 plays a pivotal role to regulate PCP in Drosophila ${ }^{5}$, as well as CE in Zebrafish ${ }^{6}$ and Xenopus. ${ }^{7}$ PRICKLE1 is an evolutionary conserved cytoplasmic protein. It contains a PET domain at the $\mathrm{N}$-terminus followed by three LIM domains and a Cterminal farnesylation site. ${ }^{8}$ Recently, we and others have demonstrated the prominent role of PRICKLE1 during cancer progression. ${ }^{2,9-11}$ PRICKLE1 is a prometastatic protein and regulates oriented cell migration in various cell lines including the MDA-MB-231 prototype TNBC cell line. ${ }^{2,10}$ At the molecular level, PRICKLE1 regulates the subcellular localisation of associated proteins such as VANGL2, ${ }^{8,12}$ RICTOR, $^{2}$ ARHGAP22 $/ 24,{ }^{10}$ and $\mathrm{LL} 5 \beta^{11}$ to coordinate oriented cellular migration.

Here, we identify the proteome associated to PRICKLE1 in MDAMB-231 cells. Among the proteins associated to PRICKLE1, our attention was drawn to a large subset of small G-protein regulators. We first show that $P R I C K L E 1$ and its associated proteins are overexpressed in TNBC, and that their upregulation is associated with poor prognosis in this molecular subtype. To further explore the protein complex associated to PRICKLE1, we focused our attention on the Rho-guanylyl exchange factor (GEF) called epithelial cell transforming sequence 2 (ECT2). In nontransformed cells, ECT2 regulates cytokinesis by regulating Rac1 activity. ${ }^{13-16} E C T 2$ is frequently upregulated in various cancers

\footnotetext{
${ }^{1}$ Centre de Recherche en Cancérologie de Marseille, Equipe labellisée 'Cell polarity, Cell Signaling and Cancer' Ligue 2018, Aix Marseille Université, Inserm, CNRS, Institut Paoli Calmettes, 13009 Marseille, France; ${ }^{2}$ Centre de Recherche en Cancérologie de Marseille, Equipe labellisée 'Predictive oncology' Ligue 2018, Aix Marseille Université, Inserm, CNRS, Institut Paoli Calmettes, 13009 Marseille, France; ${ }^{3}$ Aix Marseille Univ, CNRS, IBDM, Marseille, France and ${ }^{4}$ Centre de Recherche en Cancérologie de Marseille, Marseille Proteomics, Aix Marseille Université, Inserm, CNRS, Institut Paoli Calmettes, 13009 Marseille, France

Correspondence: Avais M. Daulat (avais.daulat@inserm.fr) or Jean-Paul Borg (jean-paul.borg@inserm.fr)

These authors contributed equally: Jean-Paul Borg, François Bertucci
}

Received: 6 December 2018 Revised: 8 March 2019 Accepted: 18 March 2019

Published online: 11 April 2019 
932

such as ovarian, ${ }^{14}$ lung, ${ }^{17}$ and breast cancers. ${ }^{18}$ ECT2 promotes Rac1 activity and increases cell growth, invasion, and tumorigenicity. ${ }^{13,16}$ Here, we show that PRICKLE1 is associated with ECT2 to regulate Rac1 activity and that Prickle 1 and Ect2 act synergistically during embryonic development. Altogether, these data demonstrate the importance of PRICKLE1 and its associated protein complex as poor-prognosis markers in TNBC and provide evidence that PRICKLE1 may be a suitable therapeutic target for treatment of this aggressive subtype of breast cancer.

\section{METHODS}

Rac1 activity assay

Cells were lysed with ice cold lysis buffer $(50 \mathrm{mM}$ Tris, $\mathrm{pH}$ 7.6, 150 $\mathrm{mM} \mathrm{NaCl}, 0.1 \%$ Triton X-100, $20 \mathrm{mM} \mathrm{MgCl}$ supplemented with protease inhibitor (Sigma)). The supernatant was collected after 10 min of centrifugation at $10,000 \times g$ at $4{ }^{\circ} \mathrm{C}$. Protein concentration was measured from the solubilised fraction and adjusted to $2 \mathrm{mg} / \mathrm{mL}$. Ten per cent of the lysates are conserved as loading controls. One hundred micrograms of GST-CRIB were added to $2 \mathrm{mg}$ of lysate and incubated with rotation during $30 \mathrm{~min}$ at $4{ }^{\circ} \mathrm{C}$. Beads were washed with 10 volumes of lysis buffer. Rac-GTP forms were eluted from the beads using $2 \times$ Laemmli buffer. Thirty per cent of the sample were ran on $15 \%$ SDS-PAGE gel and transferred to PVDF, then blotted with the indicated antibody.

Breast cancer samples and gene expression profiling Our institutional series included 353 tumour samples from pretreatment invasive primary mammary carcinomas either surgically removed or biopsied. ${ }^{19}$ Samples were profiled using Affymetrix U133 Plus 2.0 human microarrays (Santa Clara, CA, USA). The resulting data were pooled with 35 public breast cancer data sets comprising both gene expression profiles generated using DNA microarrays and RNA-Seq and clinicopathological annotations. These sets were collected from the National Center for Biotechnology Information (NCBI)/Genbank GEO, ArrayExpress, European Genome-Phenome Archive, The Cancer Genome Atlas portal (TCGA) databases, and authors' website (Supplementary Table 1). The final pooled data set included 8982 non-redundant nonmetastatic, non-inflammatory, primary, invasive breast cancers.

\section{Gene expression data analysis}

Before analysis, several steps of data processing were applied. The first step was the normalisation of each set separately. It was done in $\mathrm{R}$ using Bioconductor and associated packages; we used quantile normalisation for the available processed data from nonAffymetrix-based sets (Agilent, SweGene, and Illumina), and Robust Multichip Average (RMA) with the non-parametric quantile algorithm for the raw data from the Affymetrix-based sets. In the second step, we mapped the hybridisation probes across the different technological platforms represented as previously reported. ${ }^{20}$ When multiple probes mapped to the same GenelD, we retained the most variant probe in a particular data set. We log2-transformed the available TCGA RNA-Seq data that were already normalised. In order to avoid biases related to transinstitutional $\mathrm{IHC}$ analyses and thanks to the bimodal distribution of respective mRNA expression levels, the ER, progesterone receptor (PR), and HER2 statutes (negative/positive) were defined on transcriptional data of ESR1,PGR, and HER2, respectively, as previously described. ${ }^{21}$ The molecular subtypes of tumours were defined as HR+/HER2 - for ER-positive and/or PR-positive and HER2-negative tumours, HER2 + for HER2-positive tumours, and triple negative (TN) for ER-negative, PR-negative, and HER2negative tumours. Next, expression levels of PRICKLE1 and 10 genes of interest from the protein complex associated with Prickle1 (namely, ARHGAP21, ARGHAP22, ARHGAP23, ARHGEF2, ARHGEF40, BCR, ECT2, IQGAP3, MYO9B, and STARD13) were extracted from each of the 36 normalised data sets. Before analysis, gene expression levels were standardised within each data set using the PAM50 luminal A population as reference. This allowed to exclude biases due to laboratory-specific variations and to population heterogeneity and to make data comparable across all sets. PRICKLE1 and ECT2 upregulation in a tumour was defined by an expression level above median expression; the other cases being defined as downregulation. GEF/GAP activity was based on metagene approach and computed on the mean of the 10 related genes standardised. GEF/GAP activity "up" was defined by a metagene score value above the global median of the metagene, while a value below the global median was defined as "down".

Statistical analysis

Correlations between tumour classes and clinicopathological variables were analysed using the one-way analysis of variance (ANOVA) or the Fisher's exact test when appropriate. Metastasisfree survival (MFS) was calculated from the date of diagnosis until the date of distant relapse. Follow-up was measured from the date of diagnosis to the date of last news for event-free patients. Survivals were calculated using the Kaplan-Meier method and curves were compared with the log-rank test. The likelihood ratio (LR) tests were used to assess the prognostic information provided beyond that of PRICKLE1 model, GEF/GAP metagene or ECT2 model, assuming a $X^{2}$ distribution. Changes in the LR values (LR$\Delta X^{2}$ ) measured quantitatively the relative amount of information of one model compared with another. All statistical tests were two-sided at the $5 \%$ level of significance. Statistical analysis was done using the survival package (version 2.30) in the R software (version 2.15.2; http://www.cran.r-project.org/). We followed the reporting REcommendations for tumour MARKer prognostic studies (REMARK criteria). ${ }^{22}$

Xenopus embryo injections, plasmids, RNAs, and Mos

Eggs obtained from NASCO females were fertilised in vitro, dejellied, and cultured as described previously. ${ }^{23}$ Wild-type embryos were obtained using standard methods ${ }^{24}$ from adult animals and staged according to Nieuwkoop and Faber (1994). ${ }^{25}$ Ect2 riboprobe was generated from Xenopus laevis full-length Ect2 cDNA, obtained from Dharmacom ${ }^{\mathrm{TM}}$ (Plasmid XGC ect2 cDNA, Clone ID: 5083828; pCMV-SPORT6.ccdb). The cDNA was subcloned in $\mathrm{pBS}-\mathrm{SK}$ vector. For the Ect2 sense probe, the plasmid was linearised by Notl and transcribed with T7 RNA polymerase. For the Ect2 antisense probe, the plasmid was linearised by EcoRV and transcribed with T3 RNA polymerase. Synthetic capped mRFP mRNA was produced using Ambion mMESSAGE mMACHINE Kit. pCS2-mRFP was linearised with Notl and mRNA was synthesised with $\mathrm{Sp} 6$ polymerase. About $0.5 \mathrm{ng}$ of mRFP capped mRNA was used as injection control and tracer.

Morpholino antisense oligonucleotides (MO) were obtained from Genetools with the following: Prickle1 (Pk1) $5^{\prime}$-CCTTCTGATCCATITCCAAAGGCAT-3, ${ }^{\prime 26}$ ECT2 5'-TACTGGGAGAGCCATGTTGATTT-3'. Embryos at the two-cell stage were injected in each blastomere with various doses of MOs. Embryos were cultured in modified Barth's solution until stage 28, when they were photographed.

Extended material methods including chapter about: -Plasmid constructs and reagents, tissue culture and transfection, -Immunopurification, -Affinity purification, -immunoprecipitation and western blot, -Mass spectrometry analysis. -Protein identification, -In situ hybridisation (ISH) are available in Supplementary material and methods.

\section{RESULTS}

Mass spectrometry analysis of the PRICKLE1 complex shows that PRICKLE1 is associated with small G-protein regulators

We and others have shown that PRICKLE1 contribute to cancer cell dissemination in various cancers. ${ }^{2,9-11}$ To investigate the 


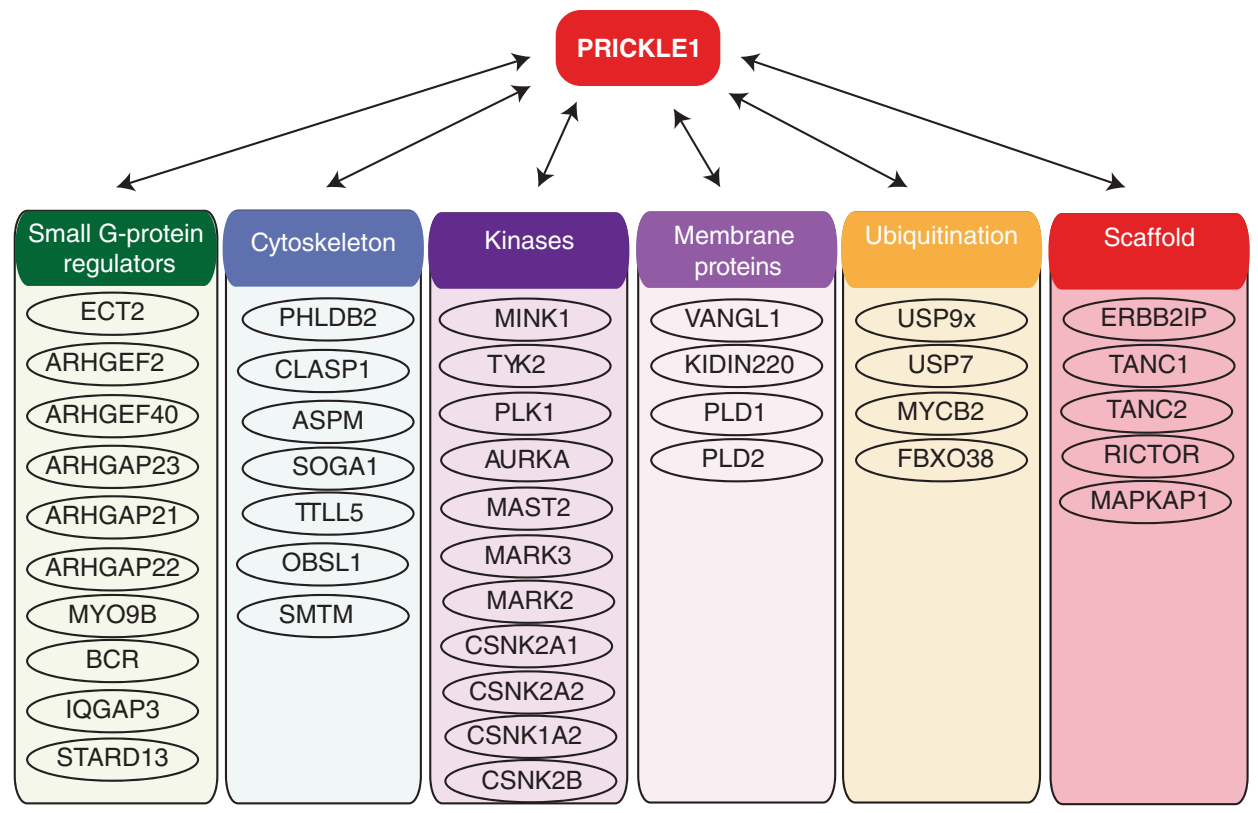

b

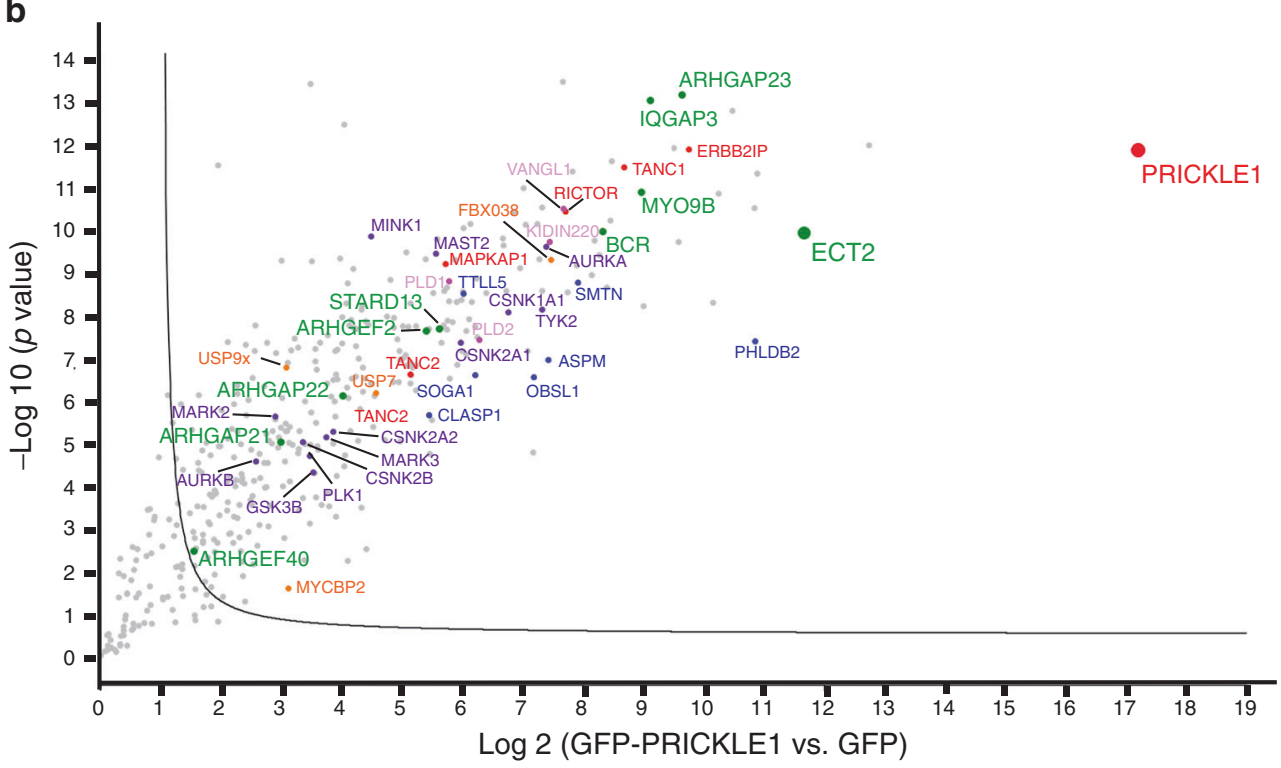

Fig. 1 Mass spectrometry analysis of the PRICKLE1 protein complex from a TNBC cell line. a Schematic representation of the proteins associated to PRICKLE1 identified by mass spectrometry analysis from MDA-MB-231 cell extracts. Proteins have been classified following their function in several groups: Small G-proteins regulators, cytoskeleton-associated, kinases, membrane proteins, proteins involved in ubiquitination, scaffold proteins, and others. b Volcano plot showing the significance two-sample $t$-test ( - Log $p$ value) vs. fold-change (Log2 (GFP-PRICKLE1 vs. GFP as control)) on the $y$ and $x$ axes, respectively. The full line is indicative of protein hits obtained at a permutation false discovery rate of $1 \%$ (pFDR). Data results from two different experiments processed three times. PRICKLE1 (the bait) is represented in red and $\mathrm{ECT} 2$, one of the most abundant PRICKLE1-associated partners, is represented in green

molecular mechanisms underlying the role of PRICKLE1 in tumorigenicity, and notably cell motility and dissemination, we generated a stable cell line expressing GFP-PRICKLE1 in the highly invasive MDA-MB-231 TNBC cell line. To identify protein complexes associated to PRICKLE1 in these cells, we performed antiGFP immunoprecipitation followed by mass spectrometry analysis. We identified previously known PRICKLE1 interactors such as VANGL1, MINK1, RICTOR, LL5 $\beta$, PLK1, and USP9x, validating our approach (Fig. 1a). Cell migration is a complex and dynamic process that involves continuous remodelling of the cellular architecture and relies on spatiotemporal modulation of signalling networks including Rho-family GTPases. Our attention was drawn to the large number of regulators of Rho-family GTPases such as Rac1, Rho, and Cdc42 (Fig. 1b), known to be involved in the regulation of cell motility, and considered as interesting drug targets to prevent cancer dissemination.

Prognostic value of PRICKLE1-interacting small G-protein regulators in TNBC

Based on these proteomic data describing the protein complex associated to PRICKLE1, we focused our attention on the 10 regulators of small G-proteins (i.e. Rho-GEF and Rho-GAP) that were identified, including ARHGAP21, ARGHAP22, ARHGAP23, ARHGEF2, ARHGEF40, BCR, ECT2, IQGAP3, MYO9B, and STARD13. 
a

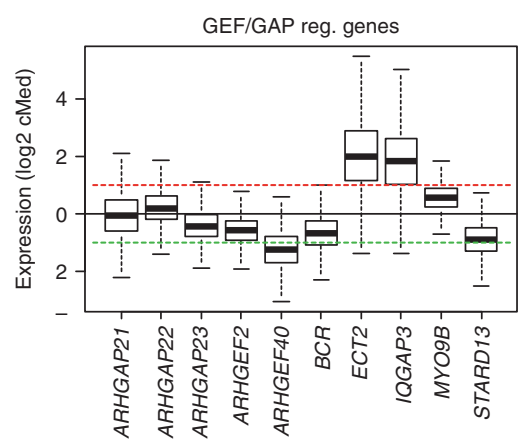

d

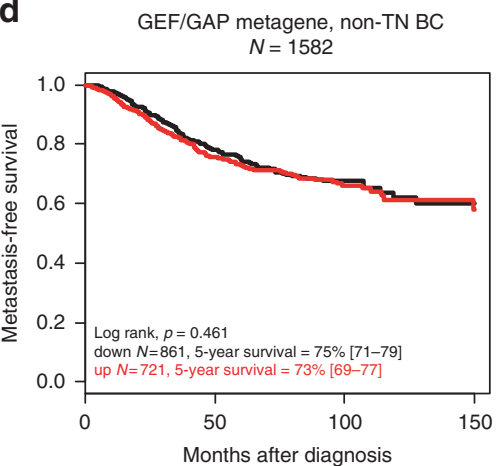

g PRICKLE1+GEF/GAP metagene, TN BC $N=449$

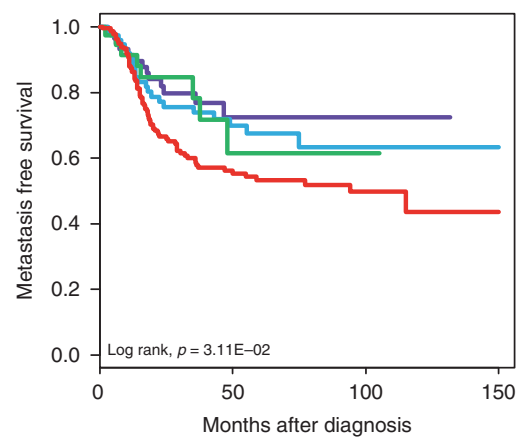

b

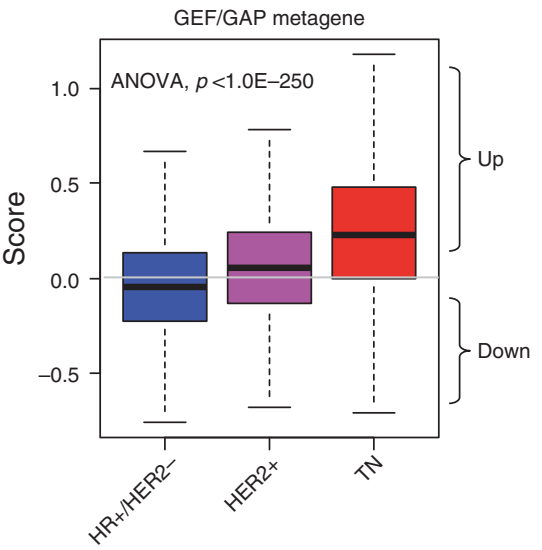

e

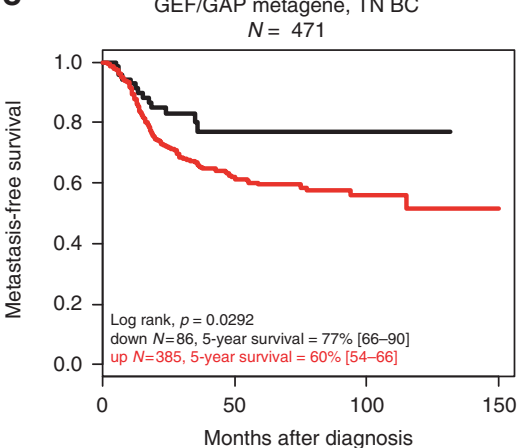

h

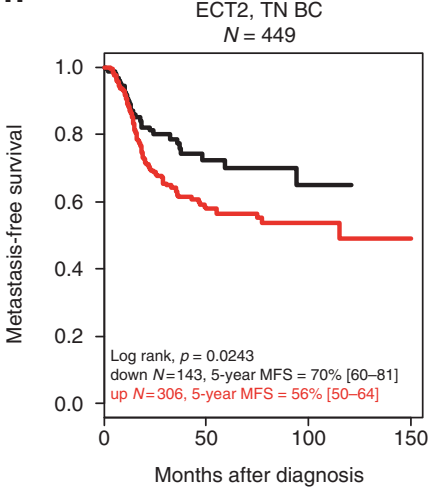

C GEF/GAP, All BC $\mathrm{N}=2030$

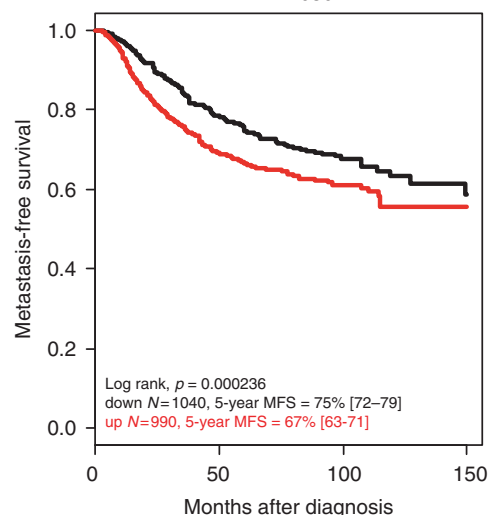

f

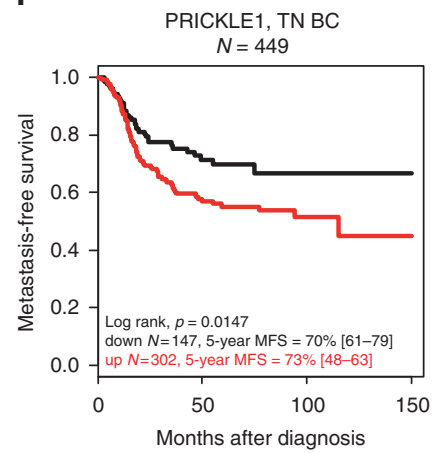

i

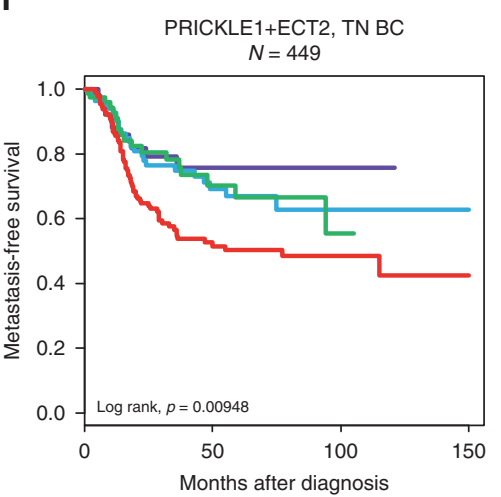

\begin{tabular}{|c|c|c|c|c|}
\hline PRICKLE1 & GEF/GAP & $n$ & 5-year MFS & $\begin{array}{c}p \text { value } \\
\text { compare to } \\
\text { down/down }\end{array}$ \\
\hline Down & Down & 63 & $72 \%$ [60-88] & \\
\hline Down & Up & 84 & $67 \%$ [57-80] & 0.495 \\
\hline Up & Down & 42 & $61 \%$ [41-92] & 0.757 \\
\hline Up & Up & 260 & $53 \%$ [46-62] & 0.0171 \\
\hline
\end{tabular}

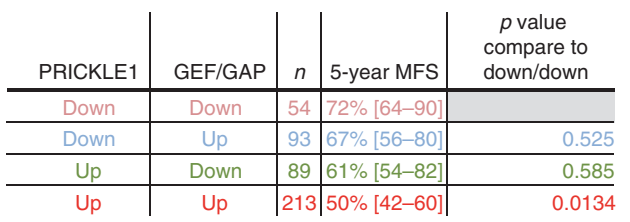

Fig. 2 Prognostic value of PRICKLE1-interacting small G-protein regulators in TNBC and cooperation between PRICKLE1 and ECT2 as poorprognosis markers. a Boxplot of GEF/GAP regulators expression across breast cancers. b Boxplot of GEF/GAP regulators expression across triple negative (TN) vs. HR+/HER2 - or HER2 + breast cancers. c Kaplan-Meier curves of metastasis-free survival among breast cancers patients according to overexpression (up) vs. underexpression (down) of GEF/GAP metagene mRNA. d Kaplan-Meier curves of metastasis-free survival among non-TNBC patients for GEF/GAP metagene mRNA expression. Kaplan-Meier curves of metastasis-free survival among TNBC patients for e GEF/GAP metagene mRNA expression, $\mathbf{f}$ PRICKLE1 mRNA expression, $\mathbf{g}$ PRICKLE1 mRNA and GEF/GAP metagene expression, $\mathbf{h}$ ECT2 mRNA expression, and i PRICKLE1 and ECT2 mRNA expression

We assessed the mRNA expression level of the corresponding genes in a retrospective series of 8982 clinically annotated patients with invasive primary breast cancer collected from several public databases (Table S1). Within these 10 genes, ECT2, $I Q G A P 3$, and MYO9B were the most overexpressed in tumours compared to normal breast tissues (Fig. 2a), whereas ARHGEF40 and STARD13 showed the lowest expression levels. We built a metagene including these 10 genes (GEF/GAP metagene) and compared its expression level in three molecular subtypes of breast cancer (HR+/HER2-, HER2+, and TN). The metagene was 
ECT2 associated to PRICKLE1 are poor-prognosis markers in triple-negative... AM Daulat et al.

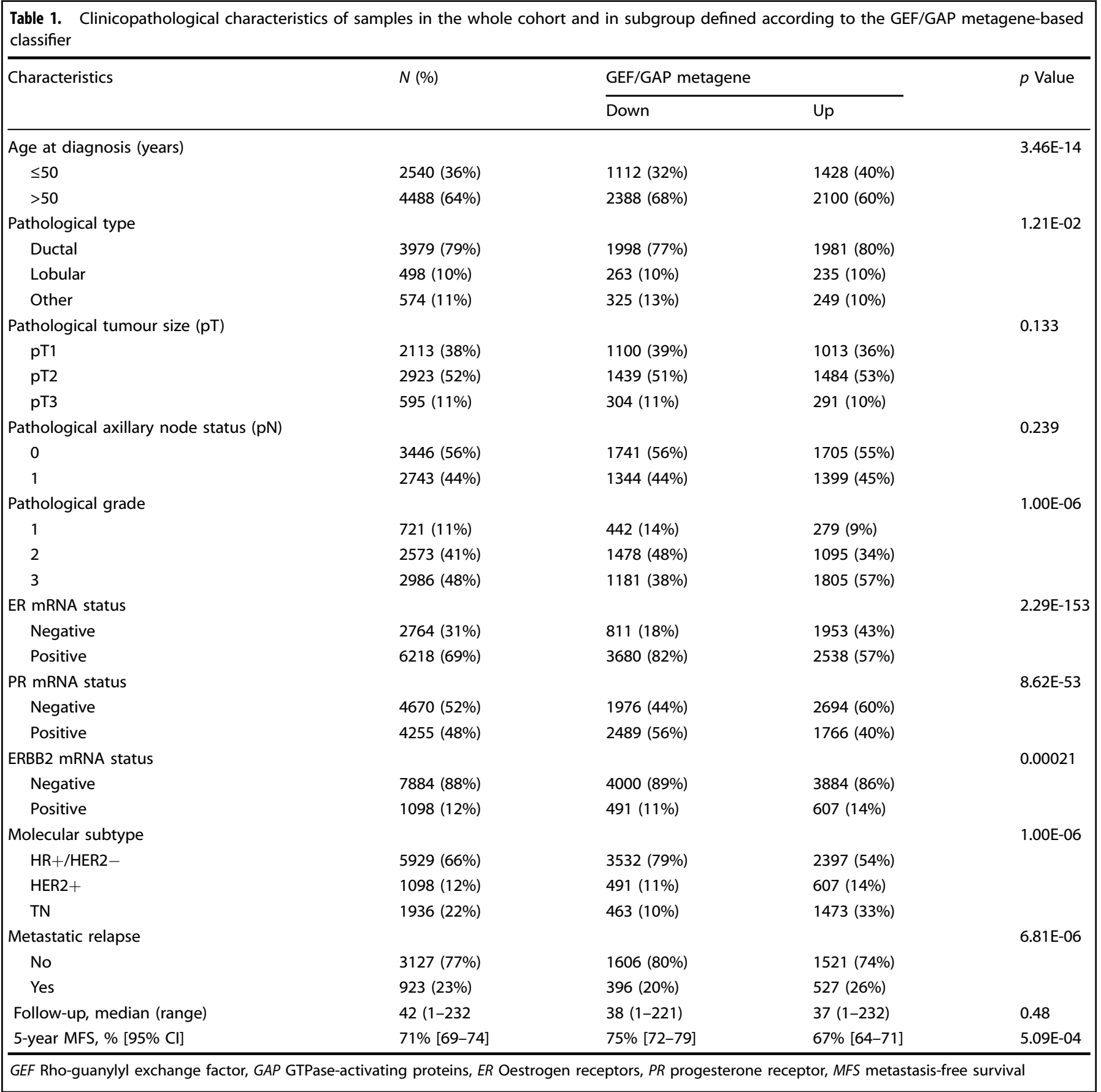

significantly upregulated in the TN subtype compared to the two other subtypes $\left(p<1.0 \times 10^{-250}\right.$, ANOVA) (Fig. 2b).

We then searched for correlations between the GEF/GAP metagene expression (as a binary variable) and the clinicopathological features of samples, including MFS. Within the 8982 breast cancer samples analysed, 4491 tumours $(50 \%)$ showed metagene upregulation when compared with normal breast (ratio $\mathrm{T} / \mathrm{NB} \geq 2$; "metagene-up" group), and 4.491 (50\%) showed metagene downregulation (ratio <2; "metagene-down" group) (Table 1). We found significant correlations between the metagene status and patients' age $(p<0.001)$, grade $(p<0.001)$, ER $(p<0.001)$, PR $(p<0.001)$, and HER2 $(p=0.012)$ statutes and with molecular subtypes of breast cancer. MFS data were available for 2030 patients: the 5-year MFS was $75 \%(95 \% \mathrm{Cl}, 72-79)$ in the "metagene-down" group vs. $67 \%(95 \% \mathrm{Cl}, 63-71)$ in the "metagene-up" group ( $p=0.00023$, log-rank test; Fig. 2c). In fact, this prognostic correlation was only observed in TNBC patients, and not in the non-TNBC ones ( $p=0.461$, log-rank test; Fig. $2 d$ ). In TNBC patients, the 5-year MFS was $77 \%(95 \% \mathrm{Cl}, 66-90)$ in the "metagene-down" group vs. $60 \%(95 \% \mathrm{Cl}, 54-66)$ in the "metagene-up" group ( $p=0.029$, log-rank test; Fig. 2e).

Cooperation between PRICKLE1 and ECT2 as poor-prognosis markers in TNBC

We have previously shown that PRICKLE1 upregulation is associated with poor MFS in basal breast cancer, ${ }^{2}$ a molecular subtype mainly composed of TNBC. In the present series of TNBC, we confirmed that PRICKLE1 upregulation was associated with 


\begin{tabular}{|c|c|c|c|}
\hline \multicolumn{4}{|l|}{ A PRICKLE1 \& GEF/GAP } \\
\hline MFS,TN BC & Statistic & & $p$ value \\
\hline PRICKLE1 & $\mathrm{LRX}^{2}$ & 6.23 & $1.25 \mathrm{E}-02$ \\
\hline PRICKLE1 + GEF/GAP & $\mathrm{LRX}^{2}$ & 8.98 & $1.12 \mathrm{E}-02$ \\
\hline GEF/GAP + PRICKLE1 vs. PRICKLE1 & $\Delta \mathrm{LRX}^{2}$ & 2.75 & 0.097 \\
\hline \multicolumn{4}{|l|}{ B PRICKLE1 \& ECT2 } \\
\hline MFS,TN BC & Statistic & & $p$ value \\
\hline PRICKLE1 & $\mathrm{LRX}^{2}$ & 6.23 & $1.25 \mathrm{E}-02$ \\
\hline PRICKLE1 + ECT2 & $\mathrm{LRX}^{2}$ & 11 & 4.14E-03 \\
\hline ECT2 + PRICKLE1 vs. PRICKLE1 & $\Delta \mathrm{LRX}^{2}$ & 4.74 & $2.90 \mathrm{E}-02$ \\
\hline
\end{tabular}

shorter MFS, with 70\% 5-year MFS (95\% Cl, 61-79) vs. 55\% (95\% Cl, 48-63) in the PRICKLE1-down group and the PRICKLE1-up group, respectively ( $p=0.0147$, log-rank test) (Fig. 2f). Since PRICKLE1 and the 10 genes of the metagene interact together, we tested whether their interaction had any prognostic value. First, we analysed the combination of the metagene expression and PRICKLE1 expression. Interestingly, patients with upregulation of both markers displayed shorter 5-year MFS (53\%, 95\% Cl, 46-62) than patients without upregulation of both markers $(72 \%, 95 \% \mathrm{Cl}$ $60-88 ; p=0.017$, log-rank test), whereas patients with intermediate status (upregulation and downregulation, and vice versa) showed intermediate 5-year MFS not significantly different from the same patients ( $p=0.757$ and $p=0.495$, respectively, log-rank test; Fig. 2g). These data suggest that metagene expression and PRICKLE1 expression might provide a complementary prognostic value. This complementarity between the two prognostic variables was tested in TNBC patients using the likelihood ratio (LR) test. As shown in Table $2 a$, the metagene added prognostic information to that provided by PRICKLE1 expression (LR- $\triangle X^{2}=2.75, p=0.097$ ).

Second, because ECT2 was one of the most prominent hit identified by mass spectrometry analysis (Fig. 1b) and the gene most overexpressed in TNBCs among members of the metagene (Fig. 2a), we investigated whether ECT2 expression alone (without the nine other genes of the metagene) would be sufficient to improve the prognostic value of PRICKLE1 expression in TNBC patients. As shown in Fig. $2 \mathrm{~h}$, patients with ECT2 upregulation displayed shorter 5 -year MFS $(56 \%, 95 \% \mathrm{Cl} 50-64)$ than patients without upregulation $(70 \%, 95 \% \mathrm{Cl} 60-81 ; p=0.0243$, log-rank test). More interestingly, ECT2 expression status increased the prognostic value of PRICKLE1 expression when combined. Patients with upregulation of both genes displayed 50\% 5-year MFS (95\% $\mathrm{Cl}, 46-62$ ) vs. $67 \%$ for patients with intermediate status (up and down, and vice-versa) vs. $76 \%(95 \% \mathrm{Cl}, 64-90)$ for patients without upregulation of both markers ( $p=0.0134$, log-rank test; Fig. 2i). The model comparison (Table $2 \mathrm{~b}$ ) showed that such ECT2 prognostic information added to that of PRICKLE1 expression was statistically significant (LR- $\Delta X^{2}=4.74, p=0.029$ ), indicating that ECT2 expression improved the prognostic value of PRICKLE1 expression in TNBC.

PRICKLE1 binds to ECT2 through its PET domain and modulates Rac1 activity

We then investigated the molecular mechanisms potentially associated to this cooperation of PRICKLE1 and ECT2 expressions to confer poor prognosis. ECT2 is a Rho-GEF and acts by exchanging GDP to GTP on the small GTPases, RhoA, Rac1, and Cdc42. ${ }^{27}$ To confirm our mass spectrometry analysis, we immunoprecipitated GFP-PRICKLE1 stably expressed in MDA-MB231 cells using GFP-targeted antibody and assessed the presence of ECT2 associated to PRICKLE1 by western blot analysis complex (Fig. 3a). We confirmed that ECT2 is associated with PRICKLE1 in MDA-MB-231 cells. We further showed that ECT2 colocalises in actin-enriched structures of lamellipodia along with PRICKLE1 using MDA-MB-231 stably expressing GFP-PRICKLE1 (Fig. 3b). We next mapped the domain of interaction between PRICKLE1 and ECT2. We thus generated deleted versions of PRICKLE1 that lack the PET and/or the LIM domains and a construct encompassing the PRICKLE1 C-terminal region. We co-transfected HEK293T cells with the indicated FLAG tagged PRICKLE1 mutants with mCherryECT2. After FLAG immunoprecipitation, we assessed the presence of mCherry-ECT2 by western blot analysis. We observed that the PET domain of PRICKLE1 was required for the formation of the PRICKLE1-ECT2 protein complex (Fig. 3c).

We further assessed PRICKLE1 contribution to Rac activity. We used previously characterised siRNAs ${ }^{2}$ to specifically downregulate PRICKLE1 expression in MDA-MB-231 cells. We observed that PRICKLE1 modulated Rac1 activity, suggesting a prominent role of PRICKLE1 in the regulation of Rho-GEF and Rho-GAP (Fig. 3d). We next set up an assay to monitor the role of PRICKLE1 on ECT2 RhoGEF activity. We expressed mCherry-ECT2 in HEK293T cells and observed an increase of active Rac1 (lane 2). However, when FLAG-PRICKLE1 was co-expressed with mCherry-ECT2, we observed an inhibitory effect of PRICKLE1 (lane 3). This observation was confirmed by the co-expression of a PRICKLE1 mutant lacking the PET and LIM1 domains which was unable to bind ECT2 and did not affect the gain of activity of ECT2 in our system (Fig. 3e, lane 4). Altogether, our data suggest that PRICKLE1 is associated with ECT2 in actin-rich structures within the lamellipodia of the cells in order to modulate the activity of ECT2 on Rac1.

Prickle1 and Ect2 functionally interact in Xenopus during embryonic development

PRICKLE1 is an evolutionarily conserved protein and plays a pivotal role during gastrulation to modulate CE movements, which are crucial to shape the body plan., ${ }^{78}$ To test whether Ect2 is required for the previously characterised function of Prickle1 during $C E$, we first compared and analysed the RNA-seq profile of prickle1 and ect2 reported on the public XenBase repository ${ }^{29}$ (data not shown). We noticed a sharp peak of zygotic ect2 expression at stage 9 , which decreases abruptly at stage 10 , just before gastrulation and CE movements take place. Zygotic prickle1 expression also begins to increase at stage 9 , reaching a maximum at stage 12 (mid gastrula), and gradually decreasing until the end of neurulation. We next performed in situ hybridisation and detected expression of ect2 RNA in the animal hemisphere up until stage 9 (Fig. 4a). Thus, ect2 transcription appears to terminate when prickle 1 transcription starts. However, inspection of genomewide proteomic data ${ }^{30}$ indicated that Ect2 protein levels were maintained during gastrulation, suggesting that Ect2 could cooperate with Prickle1 to regulate morphogenetic movements. To test this hypothesis, we performed Prickle1 and Ect2 knockdown through antisense morpholinos (MO) injections and assessed CE problems (Fig. 4b). Injection of $40 \mathrm{ng}$ Prickle1 MOs led to CE defects in $73 \%$ of embryos, in comparison to noninjected embryos (98\%) or embryos injected with RFP as control (83\%). These data are consistent with previously published results. ${ }^{7,12}$ We then injected $20 \mathrm{ng}$ of MO targeting Ect2 and we observed CE problems at a rate of $71 \%$, phenocopying the effect observed with Prickle1 MOs with narrower and shorter embryos at tailbud stage 28. We then defined subthreshold doses of individual Mo-Prickle1 ( $\leq 10 \mathrm{ng}$ ) and Mo-Ect2 ( $\leq 10 \mathrm{ng}$ ) that yielded moderate CE defects in this assay when injected separately into two blastomeres at two-cell stage (18\% and $12 \%$ CE defects, respectively). In contrast, co-injecting both MOs at subthreshold doses caused strong disruption of CE movements (67\%), suggesting that Prickle1 and Ect2 functionally interact during Xenopus embryonic development. 
a

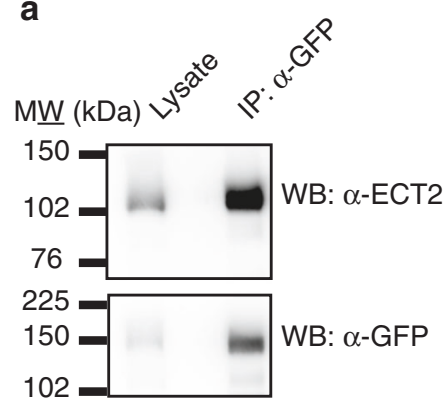

b
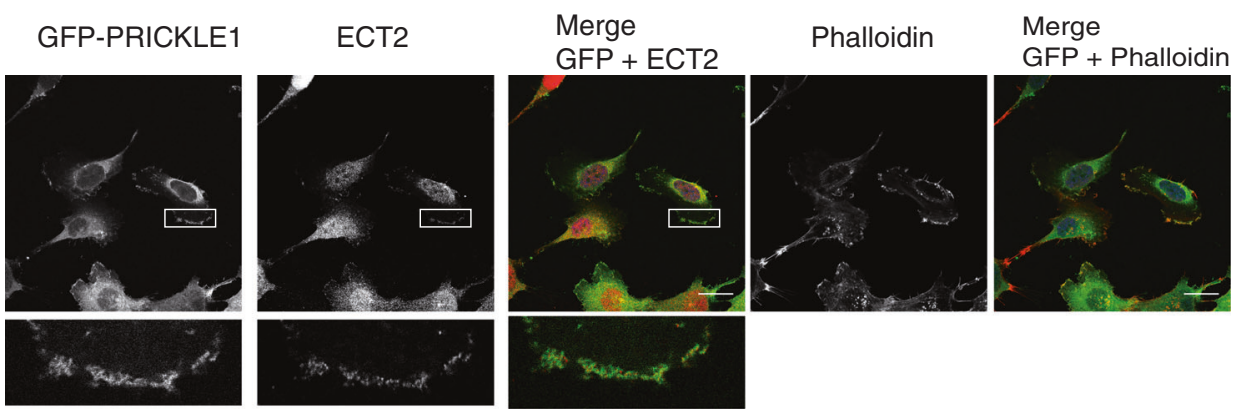

C

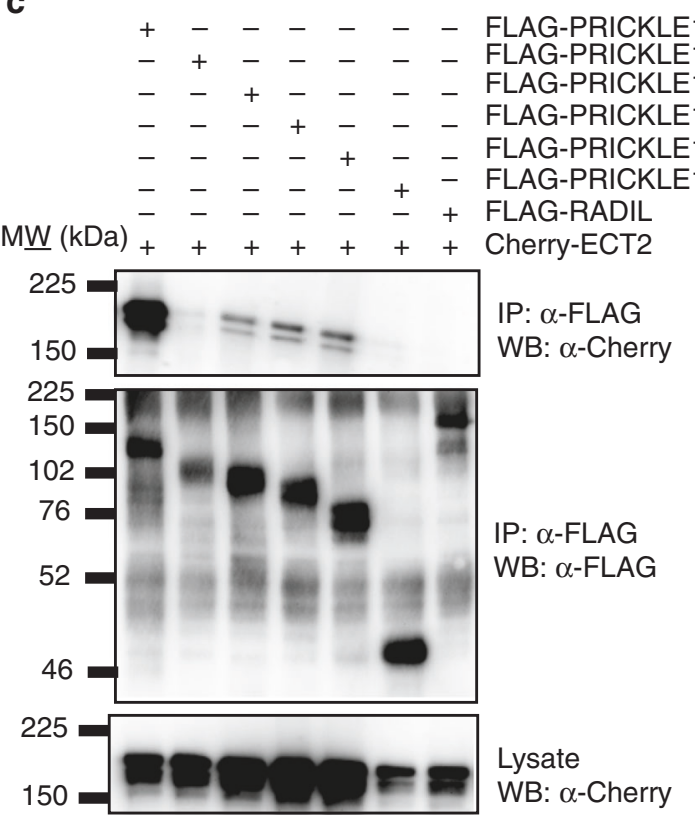

d

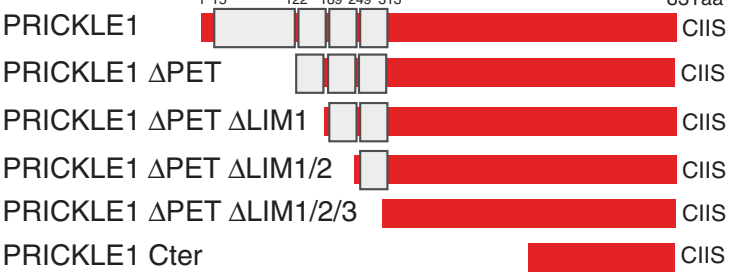

e

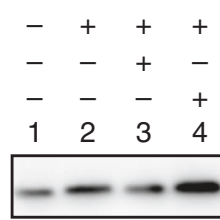

Cherry-ECT2

FLAG-PRICKLE1

FLAG-PRICKLE1 $\triangle$ PET $\Delta$ LIM1

PD: GST-CRIB

WB: $\alpha-$ Rac1

Ratio

$\begin{array}{llll}1 & 1.6 & 0.6 & 1.8\end{array}$ Rac1-GTP/Rac1 total

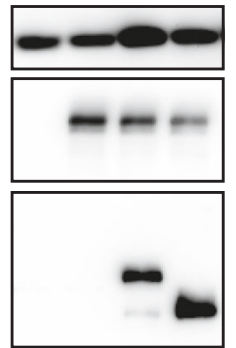

Lysate

WB: $\alpha-R a c 1$

WB: $\alpha$-Cherry (ECT2)

WB: $\alpha-$ FLAG (Prickle1 mutants)

PD: GST-CRIB

WB: $\alpha-$ Rac 1

$\begin{array}{llll}1 & 2.9 & 2.9 & \text { Ratio }\end{array}$

Rac1-GTP/Rac1 total

Lysate

WB: $\alpha-$ Rac1

Fig. 3 PRICKLE1 is associated to the Rho-GEF ECT2 and controls its activity. a Immunopurification of GFP-PRICKLE1 from MDA-MB-231 cell lysate using GFP nanobodies coupled to sepharose beads allows the identification of ECT2 associated to PRICKLE1. $\mathbf{b}$ Immunofluorescence of MDA-MB-231 cells stably expressing GFP-PRICKLE1 shows that ECT2 (endogenous) is colocalised with PRICKLE1 and enriched in actin structures within the lamellipodia. c Mapping of the PRICKLE1 domain needed for interaction with ECT2. HEK293T cells were co-transfected with the indicated forms of PRICKLE1 (see on the left for topology details) and mCherry-ECT2. After FLAG immunopurification, presence of ECT2 is detected using anti-mCherry antibody. $\mathbf{d}$ Downregulation of PRICKLE1 expression using siRNA targeting PRICKLE1 shows an increase of Rac activity in MDA-MB-231 cells. e PRICKLE1 modulates ECT2 activity. Using HEK293T cells, we expressed or co-expressed ECT2 with full length or a deleted version of PRICKLE1 lacking its domain of interaction with ECT2. Overexpression of ECT2 leads to an increase in Rac activity which was inhibited when PRICKLE1 is co-expressed. Co-expression of a mutant form of PRICKLE1 did not modify the gain of function observed by ECT2 overexpression

\section{DISCUSSION}

We and others have previously demonstrated the prominent role of PRICKLE1 during cancer progression. ${ }^{2,9-11}$ In this study, we identified the protein complex associated to PRICKLE1 and we evaluated the impact of PRICKLE1 and its associated protein complex in TNBC. Our results show that PRICKLE1 acts as a scaffold protein due to the large number of associated proteins with enzymatic activity. Among the PRICKLE1-associated proteins, we 

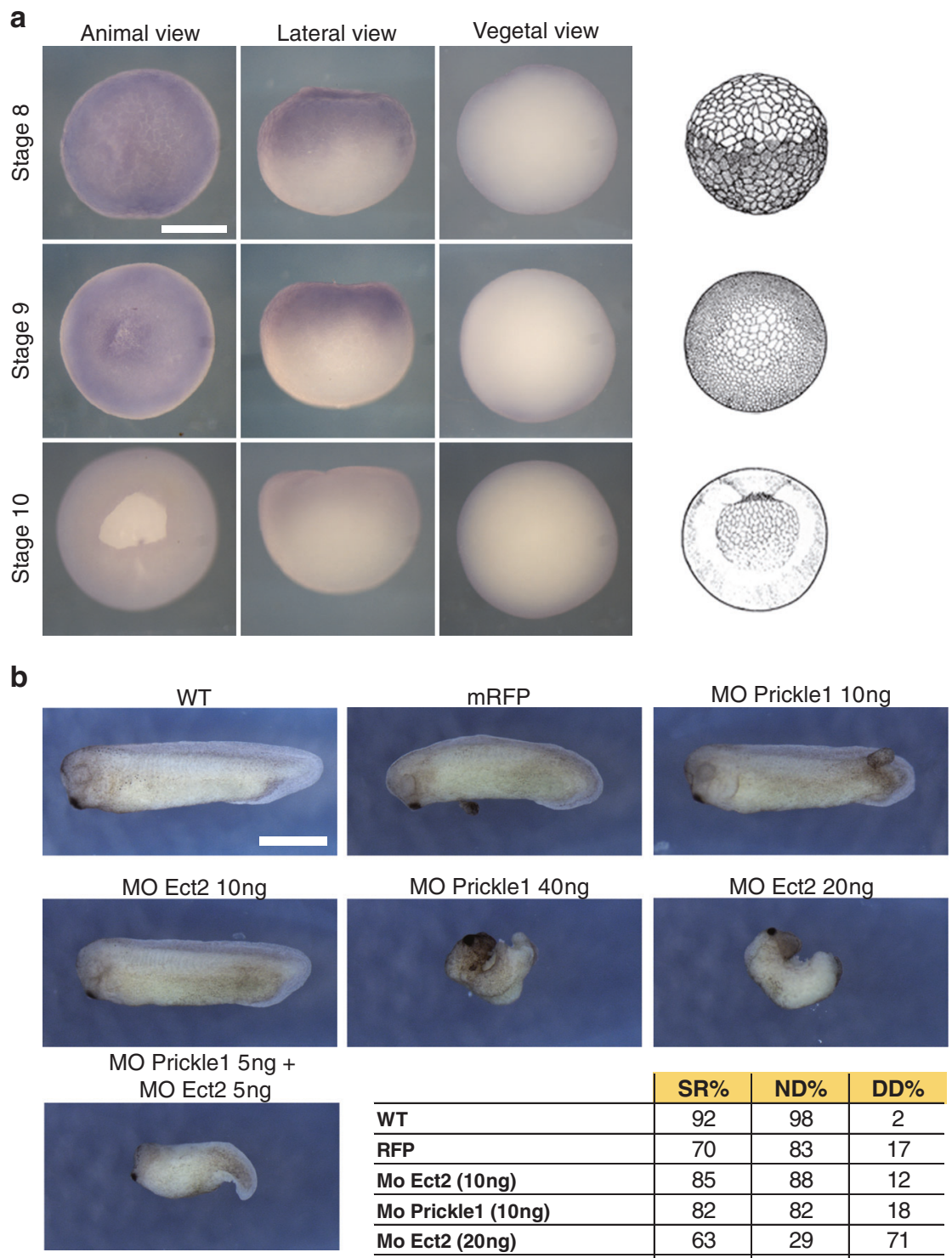

\begin{tabular}{l|c|c|c} 
& SR\% & ND\% & DD \% \\
\hline WT & 92 & 98 & 2 \\
\hline RFP & 70 & 83 & 17 \\
\hline Mo Ect2 (10ng) & 85 & 88 & 12 \\
\hline Mo Prickle1 (10ng) & 82 & 82 & 18 \\
\hline Mo Ect2 (20ng) & 63 & 29 & 71 \\
\hline Mo Prickle1 (40ng) & 50 & 27 & 73 \\
\hline Mo Ect2 + Prickle1 (5ng+5ng) & 45 & 33 & 67
\end{tabular}

Fig. 4 Prickle1 and Ect2 functionally interact in Xenopus during embryonic development. In situ hybridisation against ect2 transcripts at stage 8,9 , and 10. ect2 RNA is detectable in the animal pole (animal view and lateral view) but not in the vegetal pole (vegetal view) at stages 8 and 9 , but no longer at stage 10 . Schematic representations of embryos at the stages analysed are shown on the right. b Embryos at two-cell stage were injected into two blastomeres with Prickle1 and Ect2 MOs as indicated. In all cases $0.5 \mathrm{ng}$ of mRFP mRNA was injected as control and tracer. Suboptimal doses (10 ng) of either MO did not cause CE problems. However, when both Prickle1 and Ect2 MOs were co-injected at suboptimal doses ( $5 \mathrm{ng}$ each), embryos displayed CE problems at a rate comparable to high doses of each MO injected separately (40 ng Prickle1-MO or $20 \mathrm{ng}$ Ect2-MO). A total of 60 embryos per condition was analysed in two independent experiments. Pictures illustrate representative phenotypes. SR survival rate, ND percentage of surviving embryos developing normally, CED, percentage of surviving embryos showing convergent-extension defects. Scale bars: $\mathbf{a}=0.25 \mathrm{~mm} ; \mathbf{b}=0.5 \mathrm{~mm}$

focused our attention on small G-protein regulators since their impact on cell motility and cancer cell dissemination has been well characterised. ${ }^{31-33}$ Exploiting our transcriptomic breast cancer database, we showed that this subset of genes is upregulated in TNBC. Among this group of genes, we identified $E C T 2$ as the most prominent contributor to PRICKLE1 prognostic value. Indeed, TNBC patient with upregulated expression of both PRICKLE1 and ECT2 expression had a shorter MFS than other patients. We further characterised the PRICKLE1 and ECT2 interaction and showed that PRICKLE1 controls ECT2 function on Rac1 activation. We finally defined that Prickle1 and Ect2 interaction was evolutionary conserved, since both proteins contribute to Xenopus embryonic development and are involved in CE movements.

Among breast cancers, TNBC are considered the most aggressive form and no targeted therapy is currently available due to a lack of specific targets. ${ }^{1}$ Here, we show that PRICKLE1 is overexpressed in TNBC and is a poor-prognosis marker. PRICKLE1 is a protein highly regulated by post-translational modifications, particularly through ubiquitination/deubiquitination. PRICKLE1 is indeed the target of SMURF1, an ubiquitin ligase, which allows its rapid degradation, ${ }^{34}$ but is also protected from degradation by USP9 $x$ which de-ubiquitinates the protein. ${ }^{35}$ Interestingly USP9x is also upregulated in several cancers and is considered as a poor- 
prognosis marker. ${ }^{36}$ PRICKLE1 is also regulated through phosphorylation by the serine/threonine kinase called MINK1, which promotes its function, its membrane localisation and association with signalling molecules. ${ }^{12}$ Together, this shows that PRICKLE1 is a pivotal protein in cancer cell dissemination and a candidate target for setting up novel therapeutic strategies.

During developmental processes and cancer progression, PRICKLE1 is required for oriented cell migration. ${ }^{2,9,11,37}$ At the molecular level, we and others have shown that PRICKLE1 functions to localise VANGL at the plasma membrane, ${ }^{8,12}$ LL5 $\beta$ at the + ends of the microtubules, ${ }^{11}$ and to restrict localisation of Rho-GAP at the edge of the migrating cancer cells. ${ }^{10}$ PRICKLE1 also regulates spatial localisation of several active proteins such as mTORC2 to allow local activation of Akt at the leading edge of migrating cells, ${ }^{2}$ PHLDB2 to disassemble focal adhesions ${ }^{11}$ and to restrict RhoA activity by regulating subcellular localisation of RhoGAP. ${ }^{10}$ Together the contribution of PRICKLE1 to localisation of its interacting partners allows the cells to coordinate cellular movements and promote directed cell migration. Here we show that PRICKLE1 also contributes to regulating the activity of ECT2, a GEF for Rac1, which is essential for cell motility.

ECT2 is a Rho-GEF controlling Rac1 activity. ${ }^{13}$ Although ECT2 has been extensively studied for its role in the nucleus and during cytokinesis, reports have shown that ECT2 can also be localised in the cytoplasm of cancerous cells. ${ }^{16}$ We observed that ECT2 is localised in actin-rich structures within the lamellipodia. As described for other PRICKLE1 interactors, PRICKLE1 might contribute to ECT2 spatial localisation in order to modulate its Rac activity. Moreover, our data show that overexpression of ECT2 in HEK293T cells contributes to an increase of Rac activity, and that PRICKLE1 overexpression leads to a decrease of this gain of function, suggesting an inhibitory role of PRICKLE1 on ECT2 activity. Altogether, this depicts PRICKLE1 as a master regulator of localised expression and regulation of signalling events in migratory cancer cells.

Our data also identified a role for the PET domain of PRICKLE1, as ECT2 is to date the only protein identified to be associated with this domain. At the molecular level, it has been shown that PRICKLE1 exists in an open and closed conformation. ${ }^{38}$ It has been suggested that in the closed conformation, the three LIM domains of PRICKLE1 mask the PRICKLE1 PET domain. In an open conformation, the PET domain is unmasked, thus activating PRICKLE1. We can speculate that the interaction between PRICKLE1 and ECT2 can be modulated by switching between these two conformations as a molecular mechanism for PRICKLE1 activation.

Finally, our study identified that ECT2 is required for Xenopus embryonic development. Prickle1 has been extensively characterised for its contribution during $\mathrm{CE}^{6,7}$ movements and has been shown to be asymmetrically distributed within the cells in order to organise their movement. ${ }^{39,40}$ A previous study indicated that Prickle1 mRNA accumulates within the blastopore lip from the onset of gastrulation. ${ }^{41}$ Here, we show that ect2 mRNA and presumably Ect2 protein are expressed prior to and in a broader pattern than Prickle1. ${ }^{41}$ Knockdown experiments strongly suggest that Prickle1 and Ect2 act together to allow convergenceextension movements during gastrulation. Altogether, our data support the view that Ect2 might represent a permissive factor for Prickle1 activity. This study demonstrates the importance of the evolutionarily conserved interaction between Prickle1 and Ect2, which appears to be reactivated during tumorigenesis to promote cancer cell dissemination and metastasis.

\section{ACKNOWLEDGEMENTS}

The authors wish to thank Valérie Ferrier for critical review of the manuscript and Emilie Baudelet for technical assistance to process protein samples for mass spectrometry analysis. This work was funded by La Ligue Nationale Contre le
Cancer (Labels Ligue JPB and DB, and fellowship to A.M.D.), Fondation de France (fellowship to A.M.D.), Fondation ARC pour la Recherche sur le Cancer (grant to J.-P. B.), INCA PLBIO INCa 9474 (fellowship to D.R.), and SIRIC (INCa-DGOS-Inserm 6038, fellowship to A.M.D.). M.S.W. was a recipient of the Science without Borders PhD program from Brazil Coordenação de Aperfeiçoamento de Pessoal de Nível Superior (CAPES). The Marseille Proteomics (IBiSA) is supported by Institut PaoliCalmettes (IPC) and Canceropôle PACA. J.-P.B. is a scholar of Institut Universitaire de France.

\section{AUTHOR CONTRIBUTIONS}

A.M.D., J.P.B., and F.B. designed the study and wrote the manuscript. A.M.D. conducted the research and performed biochemistry experiments. A.M.D., M.S.W., L. C., and S.A. performed the protein complex purification and mass spectrometry analysis. P.F. and F.B. analysed transcriptomic database. D.R. and L.K. contribute to the Xenopus experiments. D.B. contributed with key insights.

\section{ADDITIONAL INFORMATION}

Supplementary information is available for this paper at https://doi.org/10.1038/ s41416-019-0448-z.

Competing interests: The authors declare no competing interests.

Ethics approval and consent to participate: Samples of human origin and associated data were obtained from the IPC/CRCM Tumour Bank that operates under authorisation \# AC-2013-1905 granted by the French Ministry of Research. Prior to sample storing, patients had been appropriately informed and had provided written informed consent, in compliance with French and European regulations. The project was approved by our Institutional Review Board. The study was performed in accordance with the Declaration of Helsinki. All Xenopus experiments were performed following the Directive 2010/63/EU of the European parliament and of the council of 22 September 2010 on the protection of animals used for scientific purposes. Experiments on Xenopus laevis were approved by the "Direction départementale de la Protection des Populations, Pôle Alimentation, Santé Animale, Environnement, des Bouches du Rhône" (agreement number F 13055 21).

Data availability: The mass spectrometry proteomics data, including search results, will be deposited to the ProteomeXchange Consortium (www.proteomexchange. org $)^{42}$ via the PRIDE partner repository with the data set identifier PXD011253.

Note: This work is published under the standard license to publish agreement. After 12 months the work will become freely available and the license terms will switch to a Creative Commons Attribution 4.0 International (CC BY 4.0).

Publisher's note: Springer Nature remains neutral with regard to jurisdictional claims in published maps and institutional affiliations.

\section{REFERENCES}

1. Tong, C. W. S., Wu, M., Cho, W. C. S. \& To, K. K. W. Recent advances in the treatment of breast cancer. Front. Oncol. 8, 227 (2018).

2. Daulat, A. M., Bertucci, F., Audebert, S., Serge, A., Finetti, P., Josselin, E. et al. PRICKLE1 contributes to cancer cell dissemination through its interaction with mTORC2. Dev. Cell 37, 311-325 (2016).

3. Butler, M. T. \& Wallingford, J. B. Planar cell polarity in development and disease. Nat. Rev. Mol. Cell Biol. 18, 375-388 (2017).

4. Sokol, S. Y. Spatial and temporal aspects of Wnt signaling and planar cell polarity during vertebrate embryonic development. Semin. Cell Dev. Biol. 42, 78-85 (2015).

5. Gubb, D. \& Garcia-Bellido, A. A genetic analysis of the determination of cuticular polarity during development in Drosophila melanogaster. J. Embryol. Exp. Morphol. 68, 37-57 (1982).

6. Veeman, M. T., Slusarski, D. C., Kaykas, A., Louie, S. H. \& Moon, R. T. Zebrafish prickle, a modulator of noncanonical $\mathrm{Wnt} / \mathrm{Fz}$ signaling, regulates gastrulation movements. Curr. Biol. 13, 680-685 (2003).

7. Takeuchi, M., Nakabayashi, J., Sakaguchi, T., Yamamoto, T. S., Takahashi, H., Takeda, $\mathrm{H}$. et al. The prickle-related gene in vertebrates is essential for gastrulation cell movements. Curr. Biol. 13, 674-679 (2003).

8. Jenny, A., Darken, R. S., Wilson, P. A. \& Mlodzik, M. Prickle and Strabismus form a functional complex to generate a correct axis during planar cell polarity signaling. EMBO J. 22, 4409-4420 (2003). 
9. Luga, V., Zhang, L., Viloria-Petit, A. M., Ogunjimi, A. A., Inanlou, M. R., Chiu, E. et al. Exosomes mediate stromal mobilization of autocrine Wnt-PCP signaling in breast cancer cell migration. Cell 151, 1542-1556 (2012).

10. Zhang, L., Luga, V., Armitage, S. K., Musiol, M., Won, A., Yip, C. M. et al. A lateral signalling pathway coordinates shape volatility during cell migration. Nat. Commun. 7, 11714 (2016).

11. Lim, B. C., Matsumoto, S., Yamamoto, H., Mizuno, H., Kikuta, J., Ishii, M. et al. Prickle1 promotes focal adhesion disassembly in cooperation with the CLASPLL5beta complex in migrating cells. J. Cell Sci. 129, 3115-3129 (2016).

12. Daulat, A. M., Luu, O., Sing, A., Zhang, L., Wrana, J. L., McNeill, H. et al. Mink1 regulates beta-catenin-independent Wnt signaling via Prickle phosphorylation. Mol. Cell. Biol. 32, 173-185 (2012).

13. Justilien, V. \& Fields, A. P. Ect2 links the PKCiota-Par6alpha complex to Rac1 activation and cellular transformation. Oncogene 28, 3597-3607 (2009).

14. Huff, L. P., Decristo, M. J., Trembath, D., Kuan, P. F., Yim, M., Liu, J. et al. The role of Ect2 nuclear RhoGEF activity in ovarian cancer cell transformation. Genes Cancer 4, 460-475 (2013).

15. Justilien, V., Ali, S. A., Jamieson, L., Yin, N., Cox, A. D., Der, C. J. et al. Ect2dependent rRNA synthesis is required for KRAS-TRP53-driven lung adenocarcinoma. Cancer Cell 31, 256-269 (2017).

16. Justilien, V., Jameison, L., Der, C. J., Rossman, K. L. \& Fields, A. P. Oncogenic activity of Ect2 is regulated through protein kinase $\mathrm{C}$ iota-mediated phosphorylation. J. Biol. Chem. 286, 8149-8157 (2011).

17. Zhou, S., Wang, P., Su, X., Chen, J., Chen, H., Yang, H. et al. High ECT2 expression is an independent prognostic factor for poor overall survival and recurrence-free survival in non-small cell lung adenocarcinoma. PLOS ONE 12, e0187356 (2017).

18. Wang, H. K., Liang, J. F., Zheng, H. X. \& Xiao, H. Expression and prognostic significance of ECT2 in invasive breast cancer. J. Clin. Pathol. 71, 442-445 (2018).

19. Sabatier, R., Finetti, P., Adelaide, J., Guille, A., Borg, J. P., Chaffanet, M. et al. Downregulation of ECRG4, a candidate tumor suppressor gene, in human breast cancer. PLoS ONE 6, e27656 (2011).

20. Bertucci, F., Finetti, P., Viens, P. \& Birnbaum, D. EndoPredict predicts for the response to neoadjuvant chemotherapy in ER-positive, HER2-negative breast cancer. Cancer Lett. 355, 70-75 (2014).

21. Lehmann, B. D., Bauer, J. A., Chen, X., Sanders, M. E., Chakravarthy, A. B., Shyr, Y. et al. Identification of human triple-negative breast cancer subtypes and preclinical models for selection of targeted therapies. J. Clin. Invest. 121, 2750-2767 (2011).

22. McShane, L. M., Altman, D. G., Sauerbrei, W., Taube, S. E., Gion, M., Clark, G. M. et al. REporting recommendations for tumour MARKer prognostic studies (REMARK). Br. J. Cancer 93, 387-391 (2005).

23. Marchal, L., Luxardi, G., Thome, V. \& Kodjabachian, L. BMP inhibition initiates neural induction via FGF signaling and Zic genes. Proc. Natl. Acad. Sci. USA 106, 17437-17442 (2009).

24. Franco, P. G., Paganelli, A. R., Lopez, S. L. \& Carrasco, A. E. Functional association of retinoic acid and hedgehog signaling in Xenopus primary neurogenesis. Development 126, 4257-4265 (1999).

25. Nieuwkoop, P. D. \& Faber, J. Normal Table of Xenopus laevis (Daudin): A Systematical and Chronological Survey of the Development from the Fertilized Egg till the End of Metamorphosis (Garland Pub, New York, 1994).
26. Dingwell, K. S. \& Smith, J. C. Tes regulates neural crest migration and axial elongation in Xenopus. Dev. Biol. 293, 252-267 (2006).

27. Basant, A. \& Glotzer, M. Spatiotemporal regulation of RhoA during cytokinesis. Curr. Biol. 28, R570-R580 (2018).

28. Wallingford, J. B., Fraser, S. E. \& Harland, R. M. Convergent extension: the molecular control of polarized cell movement during embryonic development. Dev. Cell 2, 695-706 (2002).

29. Session, A. M., Uno, Y., Kwon, T., Chapman, J. A., Toyoda, A., Takahashi, S. et al. Genome evolution in the allotetraploid frog Xenopus laevis. Nature 538, 336-343 (2016).

30. Peshkin, L., Wuhr, M., Pearl, E., Haas, W., Freeman, R. M. Jr., Gerhart, J. C. et al. On the relationship of protein and mRNA dynamics in vertebrate embryonic development. Dev. Cell 35, 383-394 (2015).

31. Abreu-Blanco, M. T., Verboon, J. M. \& Parkhurst, S. M. Coordination of Rho family GTPase activities to orchestrate cytoskeleton responses during cell wound repair. Curr. Biol. 24, 144-155 (2014).

32. Machacek, M., Hodgson, L., Welch, C., Elliott, H., Pertz, O., Nalbant, P. et al. Coordination of Rho GTPase activities during cell protrusion. Nature 461, 99-103 (2009).

33. Cook, D. R., Rossman, K. L. \& Der, C. J. Rho guanine nucleotide exchange factors: regulators of Rho GTPase activity in development and disease. Oncogene 33, 4021-4035 (2014).

34. Narimatsu, M., Bose, R., Pye, M., Zhang, L., Miller, B., Ching, P. et al. Regulation of planar cell polarity by Smurf ubiquitin ligases. Cell 137, 295-307 (2009).

35. Paemka, L., Mahajan, V. B., Ehaideb, S. N., Skeie, J. M., Tan, M. C., Wu, S. et al. Seizures are regulated by ubiquitin-specific peptidase 9X-linked (USP9X), a deubiquitinase. PLoS Genet. 11, e1005022 (2015).

36. Murtaza, M., Jolly, L. A., Gecz, J. \& Wood, S. A. La FAM fatale: USP9X in development and disease. Cell. Mol. Life Sci. 72, 2075-2089 (2015).

37. Chiapparo, G., Lin, X., Lescroart, F., Chabab, S., Paulissen, C., Pitisci, L. et al. Mesp1 controls the speed, polarity, and directionality of cardiovascular progenitor migration. J. Cell Biol. 213, 463-477 (2016).

38. Sweede, M., Ankem, G., Chutvirasakul, B., Azurmendi, H. F., Chbeir, S., Watkins, J. et al. Structural and membrane binding properties of the prickle PET domain. Biochemistry 47, 13524-13536 (2008).

39. Ciruna, B., Jenny, A., Lee, D., Mlodzik, M. \& Schier, A. F. Planar cell polarity signalling couples cell division and morphogenesis during neurulation. Nature 439, 220-224 (2006).

40. Yin, C., Kiskowski, M., Pouille, P. A., Farge, E. \& Solnica-Krezel, L. Cooperation of polarized cell intercalations drives convergence and extension of presomitic mesoderm during zebrafish gastrulation. J. Cell Biol. 180, 221-232 (2008).

41. Wallingford, J. B., Goto, T., Keller, R. \& Harland, R. M. Cloning and expression of Xenopus Prickle, an orthologue of a Drosophila planar cell polarity gene. Mech. Dev. 116, 183-186 (2002).

42. Vizcaino, J. A., Deutsch, E. W., Wang, R., Csordas, A., Reisinger, F., Rios, D., et al. ProteomeXchange provides globally coordinated proteomics data submission and dissemination. Nat. Biotechnol. [Letter]. 32, 223-226 (2014). 